\title{
Efficacy of a Solution Composed by Verbascoside, Polyvinylpyrrolidone (PVP) and Sodium Hyaluronate in the Treatment of Chemotherapy-induced Oral Mucositis in Children With Acute Lymphoblastic Leukemia
}

\author{
Elena Bardellini, DDS, MS,* Francesca Amadori, DDS, PhD,* \\ Richard Fabian Schumacher, MD, $\dagger$ Carmelita D'Ippolito, MD, $\dagger$ \\ Fulvio Porta, MD, $\dagger$ and Alessandra Majorana, MD, DDS, FP*
}

\begin{abstract}
Summary: The aim of this study was to assess the efficacy of a solution composed by verbascoside, polyvinylpyrrolidone, and sodium hyaluronate (Mucosyte) in the treatment of chemotherapyinduced oral mucositi (OM). Patients between 5 and 18 years receiving chemotherapy for acute lymphoblastic leukemia and with OM grade 1 or 2 were randomized in group A (treated with Mucosyte, 3 mouthwashes/d per $8 \mathrm{~d}$ ) and group B (treated with placebo, ie, an inert water-based solution, 3 mouthwashes/d per $8 \mathrm{~d}$ ). The OM scoring was performed at day 1 (diagnosis of OMT0), after 3 days of treatment (T1), and at day 8 (T2). Pain was evaluated through the visual analog scale with the same timing of OM measurement. A total of 56 patients were included (28 patients per group). Group A experienced a statistically significant decline of OM at T2 $(P=0.0038)$; a statistically significant difference in pain reduction between 2 groups both at T1 and at T2 $(P<0.005)$ was observed. The use of Mucosyte mouthwashes in children with chemotherapy-induced OM may be recommended as supportive therapy.
\end{abstract}

Key Words: mucositis, child, chemotherapy, verbascoside

( J Pediatr Hematol Oncol 2016;00:000-000)

$\mathrm{O}$ ral mucositis (OM) is a common side effect of chemotherapy, especially in case of hematopoietic stem cell transplantation, due to the high-dose myeloablative chemotherapy used for conditioning regimens. ${ }^{1}$ The incidence of $\mathrm{OM}$ in the pediatric population varies from $50 \%$ up to $80 \%{ }^{2,3}$ Mucositis significantly affects the quality of life in terms of pain, ability to eat, swallow, and talk; indeed, symptoms are often of such severity as to require an interruption or curtailment of therapy. In the presence of neutropenia, mucositis predisposes to septicemia, bacteremia, and fungemia. Moreover, the presence of $\mathrm{OM}$, lengthening the hospitalization timing, is an important driver of health care costs. ${ }^{4}$ Treatment guidelines for OM were issued in 2004 and were recently updated by the Multinational Association of Supportive Care in Cancer and International Society for Oral Oncology (MASCC/ISOO). Current management of OM might comprise growth factors and cytokines, anti-

Received for publication October 28, 2015; accepted July 21, 2016. From the *Department of Pediatric Dentistry, Dental Clinic, University of Brescia; and $\dagger$ Pediatric Oncology Unit of Spedali Civili di Brescia, Brescia, Italy.

The authors declare no conflict of interest.

Reprints: Elena Bardellini, DDS, MS, Dental Clinic, P.le Spedali Civili n.1, Brescia 25133, Italy (e-mail: elena.bardellini@unibs.it).

Copyright (C) 2016 Wolters Kluwer Health, Inc. All rights reserved. inflammatory agents, anesthetics, analgesics, antimicrobial and coating agents, cryotherapy, and mucosal protectants. ${ }^{5-7}$ Although several preventive and therapeutic approaches have been evaluated, no single agent has been found to be superior. Given the side effects of pharmaceutical agents, complementary therapies are increasingly being used all over the world to especially support OM-low grade. In recent years, more attention has been focused on the role of natural drugs. ${ }^{8}$ These "natural substances" are low-molecular weight organic compounds found in plants that activate a defence system against physiological and/or environmental stress. ${ }^{9-11}$ Such compounds have antioxidant, antimicrobial, and immunomodulatory properties and derive from secondary metabolites, which belong mainly to the category of terpenes, terpenoids, alkaloids, phenols, and flavonoids. ${ }^{12-14}$ Polyphenols compound are secondary metabolites widely distributed in the plant kingdom, with many biological properties, antiallergic, antiatherogenic, anti-inflammatory, antimicrobial, and antioxidant. ${ }^{15}$ Of particular interest, in recent years, are the phenylpropanoid glycosides (also synonymous with phenylethanoid glycosides) which are water-soluble derivatives of phenylpropanoids, a large group of natural polyphenols. ${ }^{16}$ Verbascoside, a polyphenol present in many plants used for food, flavouring, and medicines like olive and mullein, shows the highest scavenger activity among phenylpropanoid glycosides tested, as well as a high antioxidant power, even in comparison with other natural phenolic compounds. ${ }^{17,18}$ In fact, it inhibits and blocks proinflammatory enzymes activity, especially of inducible nitric oxide synthase (iNOS) and cyclooxygenase-2 (COX-2). ${ }^{19}$

Recent studies have indicated that the fundamental mechanisms involved in the pathogenesis of mucositis are much more complex than direct damage to epithelium alone. Chemotherapy induces cellular damage resulting in death of the basal epithelial cells. The generation of reactive oxygen species is believed to exert a role in the initiation of mucosal injury. ${ }^{6}$

The aim of this study was to assess the efficacy of a solution composed by verbascoside, polyvinylpyrrolidone (PVP), and sodium hyaluronate (Mucosyte; Biopharm, Milan, Italy), respect of placebo, in the treatment of chemotherapy-induced OM.

\section{MATERIALS AND METHODS}

\section{Sample Selection}

The study was carried out at the Pediatric Dentistry Department of the University of Brescia and at the 
Paediatric Oncology Unit of Spedali Civili of Brescia (Italy). Consecutive patients receiving chemotherapy for acute lymphoblastic leukemia (ALL) (elaborate on the 2009 standard protocol) and with OM grade 1 or 2 were considered eligible for this study.

Enrolment criteria were: patients aged between 5 and 18 years with ALL undergoing any 1 of the phases of the chemotherapy protocol and with OM grade 1 or 2. Exclusion criteria were: OM grade $>2$, other hematologic malignancies than ALL, hypersensitivity or allergy to any of the components included in the study; radiotherapy; antibiotic and antifungal drugs assumption. Age, sex, oral hygiene grade, and absolute neutrophil count (ANC) were recorded for each patient.

\section{Study Design}

This study was designed as a double-blinded placebocontrolled study. Patients were randomized by the computer code in 2 groups: group A treated with Mucosyte (Biopharm) rinses and group B treated with placebo (ie, an inert water-based solution). The 2 products were identical regarding packaging and texture, identified only by a color label. Patients were instructed to rinse with $15 \mathrm{~mL}$ of solution (Mucosyte or placebo) for 2 minutes 3 times per day for 8 days.

Randomization was performed using an automatically generated list in a 1:1 block size for 2 patients. Patients included in the study were randomly assigned to 1 of the 2 treatments. Patients received a number after inclusion, corresponding to a color-coded mouth rinse (red for Mucosyte and blue for placebo). The list and the colored bottles were provided by the statistic while the clinical researchers made the assignation of numbers in consecutive order. Color codes were not opened until the end of the study. Both patients and researchers were blinded throughout the study.

\section{OM Scoring}

OM grade was scored by the same 2 pediatric dentists blind to the randomized allocation, according to the Common Toxicity Criteria Scale of the WHO. Lesions were classified as grade $0=$ none; grade $1=$ soreness and erythema; grade $2=$ erythema and ulcers without difficulties in swallowing solid food; grade $3=$ ulcers requiring only a liquid diet, and grade $4=$ introduction of parental nutrition.

Oral examination and $\mathrm{OM}$ scoring were performed at day 1 (diagnosis of OM) (T0), after 3 days of treatment (T1) and at day 8 (T2).

\section{PAIN Scoring}

Pain was evaluated through the visual analog scale (VAS) at the same timing of OM scoring. In this system, 1 indicates no pain and 10 indicates severe pain; patients were asked to select a number from 1 to 10 on a ruler with drawn faces to express the intensity of their pain (Wong Baker face scale). Request of additional analgesia, which means the number of times that children required pharmacological support, was also recorded.

\section{Oral Hygiene}

Clinical assessment of oral hygiene status was performed according to criteria of simplified oral hygiene index (OHI-s) by Greene and Vermillion ${ }^{20}$ with a mouth mirror and explorer.

\section{Statistical Analysis}

Statistical analysis was descriptive, including mean, $\mathrm{SD}$, and percentiles for variables as sex, age, and localization.

To compare the 2 groups, we analyzed the data on sex and disease as frequencies and percentages. Concordance or differences in the frequency distribution between the 2 groups were tested using Student $t$ test. A level of significance of $5 \%$ was used and data were analyzed using Stata software for Mac. The differences in decline of OM and VAS score were analysed using Wilcoxon rank sum test. The difference of the mean of the OHI-s and ANC at T0 and T2 was evaluated using 2 ways analysis of variance.

Taking the hypothesis that there is a success percentage of $80 \%$ at day 8 for the group treated by Mucosyte rinses and $40 \%$ for control group, the minimum number of patients for the study, assuming $\alpha=0.05$ and $\beta=0.20$ (study power $=80 \%$ ), has been calculated to be 55 (at least 23 per group).

\section{Ethical Considerations}

The study was planned and carried out in compliance with the Declaration of Helsinki and Good Clinical Practice. All patients and their caregivers were informed about the research and signed an Institutional review boardapproved informed consent.

\section{RESULTS}

A total of 59 patients were included in the study according to the enrolment criteria. Three were excluded because caregivers did not sign the informed consent. Group A (Mucosyte) was composed by 28 children (19 female and 9 male, mean age $7 \pm 1.8$ ) and group B (placebo) by 28 children (15 female and 13 male, mean age $8 \pm 2.7)$.

\section{OM Grading}

OM appeared at a mean of 6.1 days after chemotherapy (range, 4 to $8 \mathrm{~d}$ ).

At T0, the median of OM grading was 2 for group A and 2 for group B $(P>0.05)$. At the third day (T1), OM grading median was 1 for group $\mathrm{A}$ and 2 for group B $(P=0.65)$; after 8 days (T2), OM grading median resulted 0 in group $\mathrm{A}$ and 1 in group $\mathrm{B}(P=0.038)$. Group A experienced a statistically significative decline of OM just at T2 $(P=0.0038)($ Fig. 1).

\section{Pain Evaluation}

The medians of VAS at the 3 intervals and $P$-values obtained by Wilcoxon rank sum test are displayed in Table 1. A statistically significative difference in pain reduction between 2 groups both at T1 and at T2 $(P<0.05)$ was observed. During the study period, children treated by Mucosyte rinses required less additional analgesia (paracetamol, tramadol, or morphine) with respect to those belonging to the control group $(P<0.05)$.

\section{Oral Hygiene and ANC}

The mean of OHI-S at T0 and T2 in both groups was not statistically different $(P=0.15)$.

The mean of ANC at T0 and T2 in both groups was not statistically different $(P=0.63)$. 


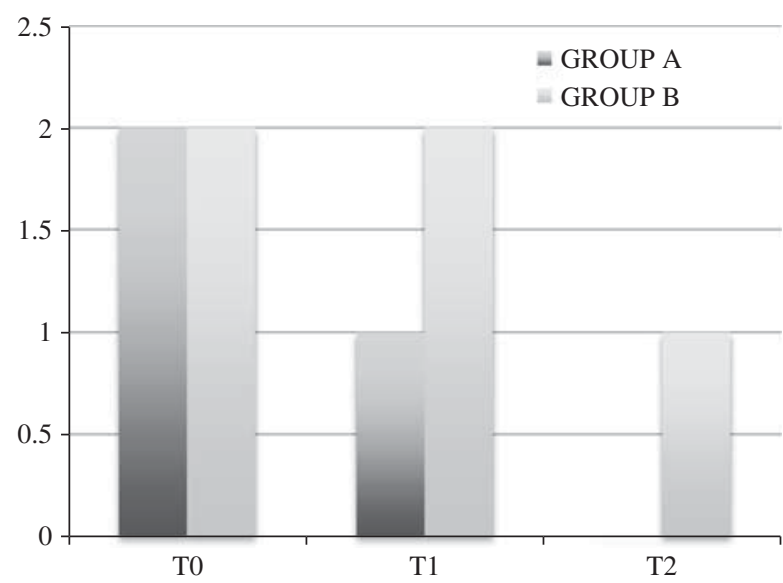

FIGURE 1. Results for decline in oral mucositis grade at T0, T1, and $\mathrm{T} 2$ in the group A and B. T0 indicates diagnosis of oral mucositis on day $1 ; \mathrm{T} 1$, after 3 days of treatment; T2, at day 8 .

\section{DISCUSSION}

OM can aggravate the pediatric patients' clinical condition and elicit multiple debilitating oral symptoms that irrevocably alter patients' quality of life. ${ }^{2}$

The pathogenesis of chemotherapy-induced OM appears to be related to oxidative stress induced by the treatment. The reactive oxygen species cause direct and indirect damage through transcription factors, such as nuclear factor-kB, that activates iNOS, and the activator protein-1, which induce the production of proinflammatory cytokines, such as tumor necrosis factor and interleukin$6^{21,22}$

This pilot study was designed to investigate the efficacy of a solution, containing sodium hyaluronate, PVP, and verbascoside, in chemotherapy-induced OM. Verbascoside is the extract of syring vulgaris and it belongs to the fenilpropanoid family, a new class of anti-inflammatory drugs.

Previous studies have suggested that verbascoside can prevent the oxidative stress as it reduces the production of superoxide radicals and consequently reduces the activity of iNOS and COX-2 [23]. Moreover, it is involved in controlling transforming growth factor beta-activated kinase, a novel protein that mediates proinflammatory signaling through induction of the transcription factor activator protein -1 . Verbascoside induces the dose-dependent decrease of the expression of interleukin-8, inhibiting proinflammatory activity of enzymes like COX-2 and iNOS, showing a cortison-like activity. ${ }^{14,23}$ Recent studies have demonstrated that sodium hyaluronate accelerates

TABLE 1. Results for Decline in Visual Analog Scale at T0, T1, and T2 in the Group A and in the Group B

\begin{tabular}{lcccc}
\hline & Group A & Group B & W (Wilcoxon test) & $\boldsymbol{P}$ \\
\hline T0 & 4 & 4 & 3805.5 & 0.90 \\
T1 & 1 & 2 & 3252 & $0.002^{*}$ \\
T2 & 0 & 1 & 3160.5 & $0.0005^{*}$ \\
\hline \multicolumn{5}{c}{ Medians, Wilcoxon rank sum test and $P$-value. Test: Wilcoxon rank sum } \\
test. \\
T0 indicates diagnosis of oral mucositis on day 1; T1, after 3 days of \\
treatment; T2, at day 8. \\
$* P<0.05$.
\end{tabular}

healing ${ }^{24}$ and helps to manage pain ${ }^{25}$ in patients with OM. Some authors stated that the compound acts as a physical barrier between the oral environment and oral mucosa, inducing also biomolecular and physiological changes in keratinocytes and mesenchimal cells. ${ }^{26,27}$

The severity of $\mathrm{OM}$ is mainly determined by the type(s) and dose of cancer therapeutic agents used. The therapeutic trend of combining different chemotherapeutic drugs further intensifies the likelihood of mucositis. ${ }^{28}$ To exclude possible confounding factors, we selected patients with ALL, undergoing the same chemotherapy protocol (ALL 2009 standard protocol), which inter alia induces a less deep and shorter aplasia. ${ }^{29}$ Instead, we did not consider the treatment stage as a variable (to evaluate the efficacy of the mouthwash) because there are still no studies that correlate OM (in terms of "duration" and "rate of healing") with the stage of treatment that caused it.

Moreover, we took into account only $\mathrm{OM}$ grade 1 or 2 , usually requiring only topical therapy, not combined with other device or drugs (ie, laser therapy, grow factors, steroids). As regards the diagnosis of OM, a concomitant herpes simplex virus infection-which is a known risk factor for aggravation of the severity of $\mathrm{OM}^{30}$ - was excluded because all patients underwent an antiviral prophylaxis $(5 \mathrm{mg} / \mathrm{kg} /$ dose 3 times a day).

The results of this investigation suggest clinical benefits in the use of the tested solution. According to our results, OM significantly reduces in the study group respect to the control group in 8 days. Also the pain, already after 3 days, is significantly decreased both from VAS results and from the request of additional analgesia. The benefit may depend on the synergic action of active components. The mucoadhesive properties of PVP allow the continuous release of sodium hyaluronate, which favors reepithelization and wound healing, and of verbascoside, which has an anti-inflammatory action.

To exclude other confounding factors, we considered also the oral hygiene grade and the ANC. The grade of oral hygiene can affect the incidence and severity of mucositis. Maintenance of good and intensified oral hygiene is a fundamental component of basic oral care. ${ }^{31}$ When oral hygiene is compromised, gingival and mucosal inflammation caused by oral bacteria increases the risk of OM up to bacteremia. ${ }^{32-34}$ According to our results, the grade of oral hygiene was not statistically different in the 2 groups. This result was expected due to the strict oral hygiene protocols to which oncohematological patients are subjected according to the international guidelines. $5,6,31$

ANC was recorded because a decrease in the neutrophil count may result in an impaired ability to protect against oral mucosal damage and may affect the proliferation of oral epithelial cells. Neutropenic patients are at increased risk for microbial colonization of damaged mucosal surfaces, resulting in increased proinflammatory cytokines in oral mucosa, which may aggravate OM.2,35,36

The ANC was similar in the 2 groups and ranged between $0.2 \times 10(9)$ and $0.5 \times 10(9) / \mathrm{L}$. So, even if the patients with $\mathrm{OM}$ tended to be neutropenic, these data did not affect the outcome of the study. The results of this pilot study should be interpreted with caution. Even if we tried to exclude confounding factors, one limitation was the small sample size. Within this limit, we can conclude that the use of Mucosyte may lead to improvements in clinical parameters in children with chemotherapy-induced low-grade OM and it may be recommended as supportive therapy. 


\section{REFERENCES}

1. Bardellini E, Schumacher F, Conti G, et al. Risk factors for oral mucositis in children receiving hematopoietic cell transplantation for primary immunodeficiences: a retrospective study. Pediatr Transplant. 2013;17:492-497.

2. Cheng KK, Molassiotis A, Chang AM, et al. Evaluation of an oral care protocol intervention in the prevention of chemotherapy-induced oral mucositis in paediatric cancer patients. Eur J Cancer. 2001;37:2056-2063.

3. Chen CF, Wang RH, Cheng SN, et al. Assessment of chemotherapy-induced oral complications in children with cancer. J Pediatr Oncol Nurs. 2004;21:33-39.

4. Scully C, Epstein J, Sonis S. Oral mucositis: a challenging complication of radiotherapy, chemotherapy, and radiochemotherapy: part 1, pathogenesis and prophylaxis of mucositis. Head Neck. 2003;25:1057-1070.

5. Lalla RV, Bowen J, Barasch A, et al. Mucositis guidelines leadership group of the Multinational Association of Supportive Care in Cancer and International Society of Oral Oncology (MASCC/ISOO). MASCC/ISOO clinical practice guidelines for the management of mucositis secondary to cancer therapy. Cancer. 2014;120:1453-1456.

6. Lalla RV, Sonis ST, Peterson DE. Management of oral mucositis in patients who have cancer. Dent Clin North Am. 2008;52:61-77.

7. Clarkson JE, Worthington HV, Furness S, et al. Interventions for treating oral mucositis for patients with cancer receiving treatment. Cochrane Database Syst Rev. 2010;4:CD001973. Doi:10.1002/14651858.CD001973.pub4.

8. Miranzadeh S, Adib-Hajbaghery M, Soleymanpoor L, et al. Effect of adding the herb Achillea millefolium on mouthwash on chemotherapy induced oral mucositis in cancer patients: a double-blind randomized controlled trial. Eur J Oncol Nurs. 2015;19:207-213.

9. Asghar Z, Masood Z. Evaluation of antioxidant properties of silymarin and its potential to inhibit peroxyl radicals in vitro. Pak J Pharm Sci. 2008;21:249-254.

10. Riccioni G, Speranza L, Pesce M, et al. Novel phytonutrient contributors to antioxidant protection against cardiovascular disease. Nutrition. 2012;28:605-610.

11. Choi JS, Chun KS, Kundu J, et al. Biochemical basis of cancer chemoprevention and/or chemotherapy with ginsenosides. Int J Mol Med. 2013;32:1227-1238.

12. Reale M, Pesce M, Priyadarshini M, et al. Mitochondria as an easy target to oxidative stress events in Parkinson's disease. CNS Neurol Disord Drug Targets. 2012;11:430-438.

13. de Cássia da Silveira e Sá R, Andrade LN, Dos Reis Barreto de Oliveira R, et al. A review on anti-inflammatory activity of phenylpropanoids found in essential oils. Molecules. 2014;19:1459-1480.

14. Speranza L, Franceschelli S, Pesce M. Anti-inflammatory properties of the plant verbascum mallophorum. J Biol Regul Homeost Agents. 2009;23:189-195.

15. Manach C, Scalbert A, Morand C, et al. Polyphenols: food sources and bioavailability. Am J Clin Nutr. 2004;79:727-747.

16. Rubio-Senent F, Lama-Munoz A, Rodriguez-Gutierrez G, et al. Isolation and identification of phenolic glucosides from thermally treated olive oil byproducts. J Agric Food Chem. 2013;61:1235-1248.

17. Grigore A, Colceru-Mihul S, Litescu S, et al. Correlation between polyphenol content and anti-inflammatory activity of verbascum phlomoides (mullein). Pharm Biol. 2013; 51:925-929.

18. Pereira JA, Pereira AP, Ferreira IC, et al. Table olives from Portugal: phenolic compounds, antioxidant potential, and antimicrobial activity. J Agric Food Chem. 2006;54:8425-8431.

19. Lee JY, Woo ER, Kang KW. Inhibition of lipopolysaccharideinducible nitric oxide synthase expression by acteoside through blocking of AP-1 activation. $J$ Ethnopharmacol. 2005;97: $561-566$.

20. Greene JC, Vermillion JR. The simplified oral hygiene index. J Am Dent Assoc. 1964;68:7-13.

21. Wong WR, Kossodo Slochevar IE. Influence of cytokines on matrix metalloproteinases produced by fibroblasts cultured in monolayer and collagen gels. J Formos Med Assoc. 2001;100: 377-382.

22. Herouy Y. Matrix metalloproteinases in skin pathology. Int $J$ Mol Med. 2001;7:3-12.

23. Speranza L, Franceschelli S, Pesce M, et al. Antiinflammatory effects in THP-1 cells treated with verbascoside. Phytother Res. 2010;24:1398-1404.

24. Favia G, Mariggio MA, Maiorano F, et al. Accelerated wound healing of oral soft tissues and angiogenic effect induced by a pool of aminoacids combined to sodium hyaluronate (AMINOGAM). J Biol Regul Homeost Agents. 2008;22:109-116.

25. Colella G, Cannavale R, Vicidomini A, et al. Efficacy of a spray compound containing a pool of collagen precursor synthetic aminoacids (1-proline, l- leucine, 1-lysine and glycine) combined with sodium hyaluronate to manage chemo/radiotherapy-induced oral mucositis: preliminary data of an open trial. Int J Immunopathol Pharmacol. 2010;23:143-151.

26. Colella G, Vicidomini A, Soro V, et al. Molecular insights into the effects of sodium hyaluronate preparations in keratinocytes. Clin Exp Dermatol. 2012;37:516-520.

27. Mariggiò MA, Cassano A, Vinella A, et al. Enhancement of fibroblast proliferation, collagen biosynthesis and production of growth factors as a result of combining sodium hyaluronate and aminoacids. Int J Immunopathol Pharmacol. 2009;22: 485-492.

28. Scully C, Epstein J, Sonis S. Oral mucositis: a challenging complication of radiotherapy, chemotherapy, and radiochemotherapy. Part 2: diagnosis and management of mucositis. Head Neck. 2004;26:77-84.

29. Morais EF, Lira JA, Macedo RA, et al. Oral manifestations resulting from chemotherapy in children with acute lymphoblastic leukemia. Braz J Otorhinolaryngol. 2014;80:78-85.

30. Santos de Faria AB, Silva IH, de Godoy Almeida R, et al. Seroprevalence of herpes virus associated with the presence and severity of oral mucositis in children diagnosed with acute lymphoid leukaemia. J Oral Pathol Med. 2014;43:298-303.

31. Elad S, Raber-Durlacher JE, Brennan MT, et al. Basic oral care for hematology-oncology patients and hematopoietic stem cell transplantation recipients: a position paper from the joint task force of the Multinational Association of Supportive Care in Cancer/International Society of Oral Oncology (MASCC/ ISOO) and the European Society for Blood and Marrow Transplantation (EBMT). Support Care Cancer. 2015;23: 223-236.

32. Kashiwazaki H, Matsushita T, Sugita J, et al. Professional oral health care reduces oral mucositis and febrile neutropenia in patients treated with allogeneic bone marrow transplantation. Support Care Cancer. 2012;20:367-373.

33. Yamagata K, Arai C, Sasaki H, et al. The effect of oral management on the severity of oral mucositis during hematopoietic SCT. Bone Marrow Transplant. 2012;47:725-730.

34. Raber-Durlacher JE, Laheij AM, Epstein JB, et al. Periodontal status and bacteremia with oral viridans streptococci and coagulase negative staphylococci in allogeneic hematopoietic stem cell transplantation recipients: a prospective observational study. Support Care Cancer. 2013;21:1621-1627.

35. Ip WY, Epstein JB, Lee V, et al. Oral mucositis in paediatric patients after chemotherapy for cancer. Hong Kong Med J. 2014;20(suppl 7):4-8.

36. Hong CH, Brennan MT, Lockhart PB. Incidence of acute oral sequelae in pediatric patients undergoing chemotherapy. A review of the literature. Pediatr Dent. 2009;31:420-425. 